\title{
Truncating and missense BMPR2 mutations differentially affect the severity of heritable pulmonary arterial hypertension
} Eric D Austin*1, John A Phillips ${ }^{1}$, Joy D Cogan ${ }^{1}$, Rizwan Hamid ${ }^{1}$, Chang $\mathrm{Yu}^{2}$, Krista C Stanton ${ }^{1}$, Charles A Phillips ${ }^{1}$, Lisa A Wheeler ${ }^{3}$, Ivan M Robbins ${ }^{3}$, John H Newman ${ }^{3}$ and James E Loyd ${ }^{3}$

Address: ${ }^{1}$ Department of Pediatrics, Vanderbilt University, Medical Center, Nashville, TN, USA, ${ }^{2}$ Department of Biostatistics, Vanderbilt University, Medical Center, Nashville, TN, USA and '3Department of Medicine, Vanderbilt University, Medical Center, Nashville, TN, USA

Email: Eric D Austin* - eric.austin@vanderbilt.edu; John A Phillips - john.phillips@vanderbilt.edu; Joy D Cogan - joy.cogan@vanderbilt.edu; Rizwan Hamid - Rizwan.hamid@vanderbilt.edu; Chang Yu - chang.yu@vanderbilt.edu; Krista C Stanton - kristastanton@yahoo.com; Charles A Phillips - charlesphillips@sgu.edu; Lisa A Wheeler - lisa.wheeler@vanderbilt.edu; Ivan M Robbins - ivan.robbins@vanderbilt.edu; John H Newman - john.newman@vanderbilt.edu; James E Loyd - jim.loyd@vanderbilt.edu

* Corresponding author

Published: 28 September 2009

Respiratory Research 2009, 10:87 doi:10.1 186/1465-992I-10-87

This article is available from: http://respiratory-research.com/content//0/I/87

(c) 2009 Austin et al; licensee BioMed Central Ltd.

This is an Open Access article distributed under the terms of the Creative Commons Attribution License (http://creativecommons.org/licenses/by/2.0), which permits unrestricted use, distribution, and reproduction in any medium, provided the original work is properly cited.
Received: 22 May 2009

Accepted: 28 September 2009

\begin{abstract}
Background: Autosomal dominant inheritance of germline mutations in the bone morphogenetic protein receptor type 2 (BMPR2) gene are a major risk factor for pulmonary arterial hypertension (PAH). While previous studies demonstrated a difference in severity between BMPR2 mutation carriers and noncarriers, it is likely disease severity is not equal among BMPR2 mutations. We hypothesized that patients with missense BMPR2 mutations have more severe disease than those with truncating mutations.
\end{abstract}

Methods: Testing for BMPR2 mutations was performed in 169 patients with PAH (125 with a family history of PAH and 44 with sporadic disease). Of the 106 patients with a detectable BMPR2 mutation, lymphocytes were available in 96 to functionally assess the nonsense-mediated decay pathway of RNA surveillance. Phenotypic characteristics were compared between BMPR2 mutation carriers and noncarriers, as well as between those carriers with a missense versus truncating mutation.

Results: While there was a statistically significant difference in age at diagnosis between carriers and noncarriers, subgroup analysis revealed this to be the case only for females. Among carriers, there was no difference in age at diagnosis, death, or survival according to exonic location of the BMPR2 mutation. However, patients with missense mutations had statistically significant younger ages at diagnosis and death, as well as shorter survival from diagnosis to death or lung transplantation than those with truncating mutations. Consistent with this data, the majority of missense mutations were penetrant prior to age 36 years, while the majority of truncating mutations were penetrant after age 36 years.

Conclusion: In this cohort, BMPR2 mutation carriers have more severe PAH disease than noncarriers, but this is only the case for females. Among carriers, patients with missense mutations that escape nonsense-mediated decay have more severe disease than those with truncating mutations. These findings suggest that treatment and prevention strategies directed specifically at BMPR2 pathway defects may need to vary according to the type of mutation. 


\section{Introduction}

Pulmonary arterial hypertension (PAH) is a devastating disease that affects people of all ages. The small pulmonary arteries are primarily affected, resulting in progressive pulmonary vascular remodeling that leads to increased pulmonary vascular resistance and right heart failure [1]. PAH may occur in a variety of clinical contexts, including as a sporadic disease known as idiopathic PAH (IPAH) and as a familial disease that typically occurs among family members who share a common genetic predisposition [2,3]. Germline mutations in the bone morphogenetic protein receptor type 2 (BMPR2) gene, a member of the transforming growth factor $\beta$ superfamily, are found in the majority of individuals $(\geq 75 \%)$ with $\mathrm{PAH}$ and a positive family history of the disease; in addition, BMPR2 mutations are found in $10-25 \%$ of cases of sporadic PAH [4-7]. BMPR2-associated PAH is an autosomal dominant disease with reduced penetrance. Therefore, the presence of a BMPR2 mutation in a patient with $\mathrm{PAH}$, regardless of family history, implies that the patient has a heritable disease thus classified as heritable PAH (HPAH).

IPAH and HPAH are histopathologically indistinguishable, and historically perceived to be clinically identical [8]. Both display marked gender disparity, with 2:1 female to male prevalence of each disease [9]. Despite their similarities, recent studies have suggested that PAH patients heterozygous for a BMPR2 mutation (carriers) have more severe disease. There is growing data to support this conclusion, as recent studies comparing HPAH patients with a BMPR2 mutation to IPAH patients showed that: (a) HPAH patients were less likely to respond to acute vasodilator testing [10,11]; (b) HPAH patients presented at a younger age and with more severe hemodynamic compromise at diagnosis $[11,12]$; (c) HPAH patients had a shorter time to death or lung transplantation. [12]

Heterozygosity for a BMPR2 mutation is neither necessary nor sufficient to cause HPAH. Disease severity among affected carriers is not uniform, and reduced penetrance ( $20 \%$ ) with variable age at both diagnosis and death suggest the importance of modifiers of disease expression [13]. Since different $B M P R 2$ gene mutations have different affects on $B M P R 2$ protein production, they are likely to cause differences in phenotype. For example, many BMPR2 mutations are truncating or terminating mutations that produce no functional protein product, due to mRNA degradation via activation of the nonsense-mediated decay (NMD) pathway [14]. NMD is a mechanism of RNA surveillance used by the cell to destroy RNA transcripts that would otherwise lead to the production of deleterious proteins. Thus, NMD may modify the phenotype caused by a mutation (Figure 1; see also comprehensive review by Neu-Yilik and Kulozik [14]). The expected result

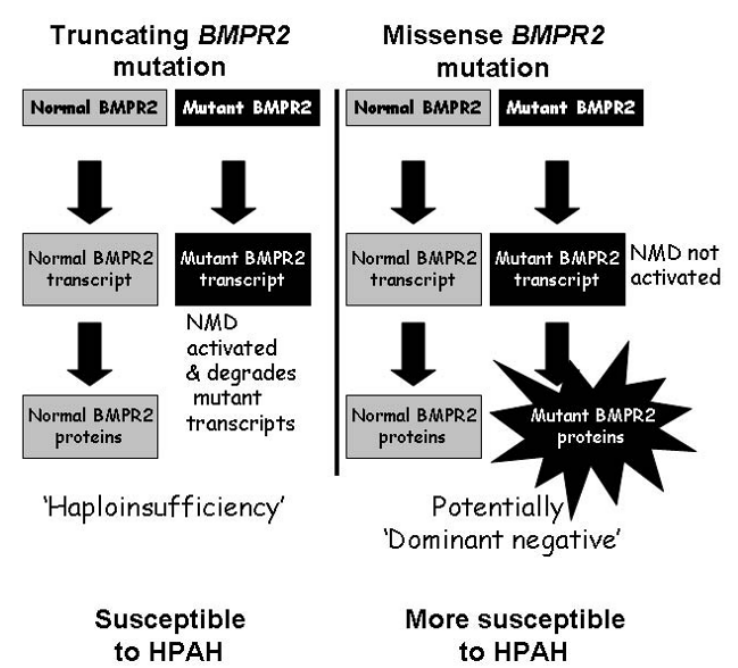

Figure I

Model of the potential impact of Nonsense-Mediated Decay (NMD) on protein expression. Activation of the NMD pathway results in the degradation of susceptible mutant transcripts, leaving only the remaining wild-type allele that produces normal BMPR2 protein. The individual may be susceptible to disease because protein amount is quantitatively but not qualitatively reduced, resulting in 'haploinsufficiency'. Mutant RNA transcripts that are resistant to NMD may result in a mutant protein with abnormal function, including the disruption of activity by normal BMPR2 protein produced by the normal allele. The potential deleterious effect of this qualitatively but not quantitatively altered protein can result in 'dominant negative' effects, and even greater susceptibility to disease.

of a mutation whose transcript is degraded by NMD is a haploinsufficient (HI) effect due to insufficient protein production, which may cause a less severe phenotype. The vast majority, but not all, of truncating mutations activate the NMD mechanism. On the other hand, a missense mutation may result in a deleterious mutated protein product that persists because it is not destroyed by NMD $[15,16]$.

While previous studies have demonstrated a difference in severity between BMPR2 mutation carriers and noncarriers, variations in severity according to BMPR2 mutation have not been explored. Wide variation in the severity of lung disease and survival is seen in other types of lung diseases due to mutations in a single gene, such as cystic fibrosis [17]. We hypothesized that the severity of HPAH could vary according to the type of BMPR2 mutation. We reasoned that carriers of a missense BMPR2 mutation that escapes NMD would have more severe disease than those who carry a truncating mutation. To test this hypothesis, the Vanderbilt Pulmonary Hypertension Research Group evaluated clinical data on all patients with PAH in whom 
genetic data were available. We found that for carriers of a truncating BMPR2 mutation, disease severity was milder and similar to that seen in noncarriers, while carriers of a missense mutation had more severe disease.

\section{Materials and methods Study Population}

The Vanderbilt Pulmonary Hypertension Research Cohort contains clinical and biologic specimens collected over 25 years, including detailed family pedigree and medical histories of patients with HPAH and IPAH. BMPR2 mutations have been detected in a large proportion of subjects tested to date. They consist of nonsense or terminating mutations that are truncating, insertion-deletion mutations that lead to splicing errors and frameshift mutations (most of which are truncating), as well as missense mutations.

One hundred and sixty-nine patients had genomic DNA available (125 with a family history of PAH and 44 with no family history) for complete BMPR2 mutation testing. One-hundred and three of the 125 with a family history were BMPR2 mutation carriers ( $82 \%)$, while 22 were noncarriers (18\%). Three of the 44 patients $(7 \%)$ with no family history and presumed IPAH were BMPR2 mutation carriers who thus had heritable disease (HPAH). Consistent with previous studies, patients from families with multiple PAH patients with no evidence of a BMPR2 mutation were excluded from the analysis to decrease the risk of misclassification in the BMPR2 noncarrier group. Circulating lymphocytes were used to derive cell lines that were used for puromycin-based NMD studies to determine the presence or absence of NMD from 96 of the 106 BMPR2 mutation carriers (Figure 2).

The majority of patients (55\%) were not diagnosed and treated at Vanderbilt University Medical Center (VUMC). For those patients not diagnosed and treated at VUMC, specialist physicians in their geographic regions identified HPAH patients, and our investigators reviewed all medical records for accuracy of diagnosis. We defined PAH diagnostically either by autopsy results showing plexogenic pulmonary arteriopathy in the absence of alternative causes such as congenital heart disease, or by clinical and cardiac catheterization criteria. These criteria included a mean pulmonary arterial pressure of more than $25 \mathrm{~mm}$ $\mathrm{Hg}$ with a pulmonary capillary or left atrial pressure of less than $15 \mathrm{~mm} \mathrm{Hg}$, and exclusion of other causes of pulmonary hypertension in accordance with accepted international standards of diagnostic criteria $[3,18]$. Clinical information concerning survival in terms of death or lung transplantation was up to date as of March 2009, the closing date for this study.

Vanderbilt Pulmonary Hypertension Research Cohort study subjects were recruited via the Vanderbilt Pulmo-

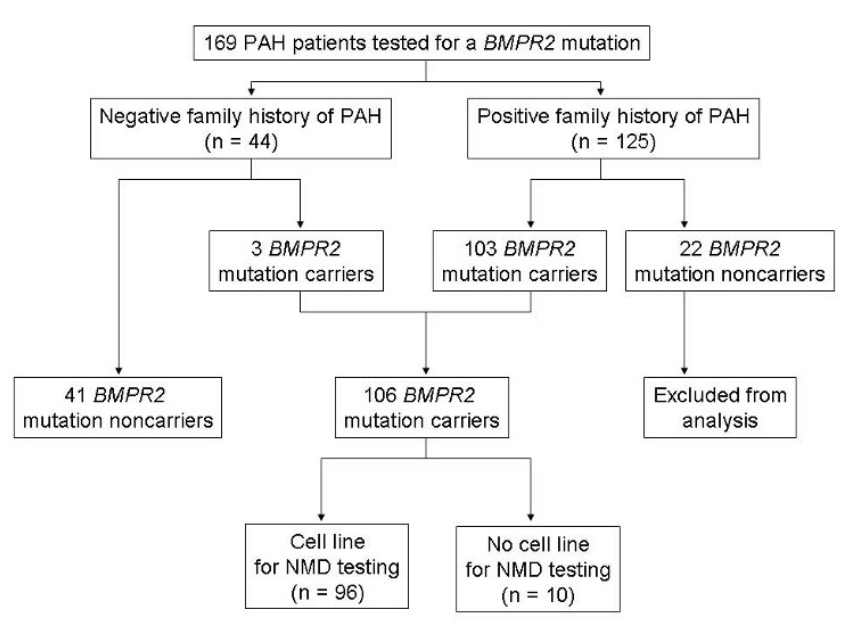

Figure 2

Study subjects. Samples from 169 consecutive subjects with PAH were tested for a BMPR2 mutation. Of these, 44 were from patients who had no family history. The remaining 125 were from patients who had a family history of PAH.

nary Hypertension Center, the Pulmonary Hypertension Association, and the NIH Clinical Trials website (http:// clinicaltrials.gov). The VUMC Institutional Review Board approved all study protocols. All participants gave informed written consent to participate in genetic and clinical studies and underwent genetic counseling in accordance with the guidelines of the American College of Chest Physicians [19]. Samples were obtained at the time of clinic visits or hospitalization or by mail via a kit for collection of whole blood and DNA.

\section{Genotyping and Genetic Analysis}

Genomic DNA was isolated from whole blood using Puregene $^{\circledast}$ DNA Purification Kits (Gentra, Minneapolis, MN) according to the manufacturer's protocol. We performed $B M P R 2$ gene mutation detection by sequencing exons and exon intron boundaries of genomic DNA and reverse transcriptase polymerase chain reaction (RT-PCR) analysis as previously described. $[20,21]$ All detected BMPR2 mutations in this study have been previously reported, and are included in a recent summary of detectable BMPR2 mutations [22].

BMPR2 gene mutations were also assessed for NMD, using protocols previously described. [16,23] Lymphocytes from each patient were incubated with and without puromycin $(250 \mu \mathrm{g} / \mathrm{ml}$, Sigma-Aldrich, St. Louis, $\mathrm{MO}$ ), which inhibits NMD, for 16 hours before harvesting. We classified mutant BMPR2 transcripts that were degraded in the absence of puromycin as NMD active if incubation with puromycin prevented this degradation. NMD active BMPR2 mutations were demonstrated to cause haploinsufficiency and classified as truncating. 
Mutations with transcripts not degraded in the absence or presence of puromycin were demonstrated to be NMD absent and these potentially dominant negative mutations were classified as missense [15].

\section{Statistical Analysis}

Demographic and clinical features were compared between BMPR2 mutation carriers and noncarriers as appropriate with the use of $\chi^{2}$ test or Fisher's exact test, and student's t test or Mann-Whitney U test. The primary study endpoint assessed was the continuous variable age at diagnosis. Because PAH can present at any age, and is characterized in some families by genetic anticipation, individuals affected at an earlier age and those with a shorter survival to death or lung transplantation may express a more severe form of disease $[12,24]$.

Kaplan-Meier survival curves were used to describe differences between groups in terms of age at diagnosis, as well as survival from diagnosis to death or lung transplantation, with comparisons made using the log-rank test. Ninety-five percent confidence intervals were reported, $P$ values less than 0.05 were considered statistically significant, and all tests were two-tailed. Statistical analyses were performed on a personal computer with the statistical package SPSS for Windows (Version 16.0, SPSS, Chicago). Of note, after careful analysis of the included subjects as well as the pedigrees from each family included in the study, it was concluded that the genetic relatedness of this cohort was not sufficient or appropriate to justify the use of family-based association tests that model genotypic risks.

\section{Results \\ Clinical Characteristics, BMPR2 Carriers versus Noncarriers}

A BMPR2 mutation was identified in 3 of 44 subjects (6.8\%) with no family history, and 103 of 125 (82.4\%) of familial cases (Figure 2). After exclusion of the 22 subjects with family history but no detectable BMPR2 mutation, 147 subjects were included in the analysis. This provided comparison of the 41 BMPR2 mutation noncarriers to the 106 carriers, who have HPAH (Table 1).

The mean age at diagnosis was significantly younger in BMPR2 mutation carriers $(\mathrm{n}=106 ; 36.1$ yrs, 95\% C.I. 33.3 to 38.8 ) compared to noncarriers $(\mathrm{n}=41 ; 42.0 \mathrm{yrs}, 95 \%$ C.I. 37.4 to 46.6$)(P=0.042$; Table 1$)$. Stratification by gender revealed that these differences were only seen among females $(\mathrm{n}=103)$, in whom the mean age at diagnosis was significantly younger in the BMPR2 mutation carriers ( $\mathrm{n}=72 ; 35.3 \mathrm{yrs}, 95 \%$ CI 31.9 to 38.7 ), as compared to noncarriers $(\mathrm{n}=31 ; 43.7$ yrs, $95 \%$ C.I. 38.5 to 48.9) $(P=0.015$; Table 1$)$. Of note, the proportion of females with a pregnancy at the time of diagnosis or
Table I: Clinical characteristics of patients at the time of diagnosis of PAH.

\begin{tabular}{llll}
\hline BMPR2 mutation & $\begin{array}{l}\text { Noncarriers } \\
(\mathbf{n}=\mathbf{4 I})\end{array}$ & $\begin{array}{l}\text { Carriers } \\
(\mathbf{n}=106)\end{array}$ & P value* \\
\hline Age at Diagnosis, yrs & $42.0(37.4-46.6)$ & $36.1(33.3-38.8)$ & 0.04 \\
Gender, female/male & $3.1 / 1$ & $2.2 / 1$ & 0.43 \\
$\mathrm{RAP}, \mathrm{mm} \mathrm{Hg}$ & $12.4(9.8-15.0)$ & $1 \mathrm{I} .1(8.9-13.3)$ & 0.29 \\
$\mathrm{Mean} \mathrm{PAP,} \mathrm{mm} \mathrm{Hg}$ & $58.3(55.0-61.5)$ & $58.6(55.3-61.8)$ & 0.97 \\
$\mathrm{PCWP}, \mathrm{mm} \mathrm{Hg}$ & $10.1(8.6-11.6)$ & $10.6(9.1-12.1)$ & 0.77 \\
$\mathrm{Cl}, \mathrm{L} / \mathrm{min} / \mathrm{m}^{2}$ & $1.8(1.4-2.2)$ & $1.9(1.7-2.0)$ & 0.92 \\
$\mathrm{PVR}, \mathrm{mm} \mathrm{Hg} / \mathrm{L} / \mathrm{min}$ & $14.0(12.4-15.5)$ & $18.1(14.1-22.0)$ & 0.08 \\
$\mathrm{~Sv}_{\mathrm{O} 2}, \%$ & $56.4(52.6-60.3)$ & $58.4(54.5-62.3)$ & 0.64 \\
\hline
\end{tabular}

* All $\mathrm{P}$ values calculated using Mann-Whitney $\mathrm{U}$ test. † $95 \%$ Confidence Interval values calculated using univariable ANOVA Definition of abbreviations: $\mathrm{Cl}=$ cardiac index; $\mathrm{PAH}=$ pulmonary arterial hypertension; PCWP = pulmonary capillary wedge pressure; $\mathrm{PAP}=$ pulmonary artery pressure; $\mathrm{PVR}=$ pulmonary vascular resistance; $\mathrm{RAP}=$ right atrial pressure; $\mathrm{Sv}_{\mathrm{O}_{2}}=$ mixed venous oxygen saturation.

within one year of diagnosis was not different between groups $(13.3 \%$ of noncarriers versus $16.7 \%$ of carriers, $P=$ $0.77)$. While fewer males were available for analysis ( $\mathrm{n}=$ $44)$, there was no difference in mean age at diagnosis (37.5 versus 36.6 years) among males.

There were no significant differences between the two groups in terms of hemodynamic characteristics at the time of diagnosis, although differences in terms of response to vasoreactivity testing were not evaluated (Table 1). Survival data were available on 138 of 147 patients studied, with 11 subjects lost to follow up. Seventy-three of the 138 patients died or underwent lung transplantation (63 BMPR2 mutation carriers and 10 noncarriers). Among those 73 patients, the 7 year difference in mean age at death/lung transplantation was not significantly different between carriers and noncarriers ( $36.6 \mathrm{yrs,}$ $95 \%$ C.I. 32.9 to 40.3 versus 43.0 yrs, $95 \%$ C.I. 31.2 to 54.7; $P=0.389$ ).

\section{Location of the BMPR2 mutations}

$B M P R 2$ gene mutations among all carriers were detected within the extracellular $(\mathrm{n}=31)$, transmembrane $(\mathrm{n}=11)$, kinase $(\mathrm{n}=40)$, and cytoplasmic $(\mathrm{n}=15)$ functional domains. One mutation was a large deletion across multiple domains, starting with the transmembrane domain. The wide distribution of mutations by location in this rare disease makes an investigation of phenotype according to location an effort that would not be expected to yield meaningful results, and a major reason that the use of nonsense-mediated decay or other means to characterize mutations phenotypically is appealing. Not surprisingly, there was no difference in age at diagnosis or death according to functional domain location of the BMPR2 mutations. In terms of their exonic locations, mutations were located across the BMPR2 gene, from exon 1 to exon 
12 , and no two families shared the same mutation. The wide variation in location for the various mutations made examining for phenotypic differences according to location statistically unsound.

\section{Clinical Characteristics, truncating versus missense BMPR2 mutations}

Of the 96 BMPR2 mutations classified regarding NMD status as truncating or missense, $55(57.3 \%)$ had a truncating mutation and $41(42.7 \%)$ had a missense mutation (Table 2; Figure 2). There was no difference according to gender, with truncating mutations found in $60 \%$ of males and $50 \%$ of females $(P=0.38)$. However, there was a significant difference in mean age at diagnosis for these subjects, with PAH diagnosed in patients with truncating mutations 10 years later than in missense mutations (truncating mutations 39.9 years (95\% C.I. 36.3 to 43.5 ) versus missense mutations 30.6 years (95\% C.I. 25.9 to 35.3); $P=0.004)$. These findings also suggested that the difference in age at diagnosis between BMPR2 carriers and noncarriers noted in this study and by previous studies was not equal for truncating and missense mutations. In fact, while there was a substantial difference between carriers with a missense mutation and noncarriers $(P=$ 0.002), there was no difference between patients with a truncating mutation and noncarriers (Figure 3). Due to limitations of sample size, comparisons between those with missense and truncating mutations were not made according to gender.

Hemodynamic parameters were not significantly different between patients with missense compared to those with truncating mutations, although differences in terms of response to vasoreactivity testing were not evaluated.

\section{Survival, truncating versus missense BMPR2 mutations}

Survival data were available on 91 of the 96 patient samples analyzed for NMD pathway activation for mutation classification. Sixty-one of the 91 patients died (31 with truncating mutations and 30 with missense mutations). The use of a systemic prostanoid medication, such as epoprostenol, was not different between groups, with this class of medication used by $68.0 \%$ of patients with a truncating mutation and $82.1 \%$ of patients with a missense mutation $(P=0.151)$. Survival was different between the two groups, with a significantly shorter time to death or lung transplantation for patients with a missense mutation compared to those with a truncating mutation (1832 days, 95\% CI 1272 to 2394 versus 3648 days, 95\% CI 2304 to 4991 ; $\log$ rank test, $P=0.044$ ) (Figure 4 ). In addition, the mean age at death/transplantation was significantly younger in the missense mutation group compared to the truncating mutation group (32.4 yrs, 95\% CI 26.9 to 38.0 versus 42.0 yrs, $95 \%$ CI 37.4 to $46.6 ; P=0.014)$.

\section{Penetrance, according to mutation type and age}

An exact evaluation of penetrance among BMPR2 mutation carriers is currently not possible due to the inherent ascertainment bias associated with the collection of subject specimens; our cohort is enriched for patients with a BMPR2 mutation compared to unaffected mutation carriers. However, due to the differences in age at diagnosis and death among patients with missense and truncating mutations, we examined disease penetrance as a function of age. Due to the differences in age at diagnosis and death among patients with missense and truncating mutations, we examined disease penetrance as a function of age. The mean age at diagnosis for the entire cohort (36 years) was chosen $a$ priori as a dividing point for this evaluation. We found a statistically significant difference in penetrance in the comparison of the missense mutation group versus the truncating mutation group $(P=0.01)$, with $68.3 \%$ of carriers of a missense mutation diagnosed prior to age 36 years, versus $41.3 \%$ of carriers of a truncating mutation (Figure 5). Of the 65 females within this analysis, 33 were diagnosed prior to age 36 years (18 within the missense versus 15 within the truncating mutation groups); 5 of the missense $(27.8 \%)$ versus 4 of the truncating $(26.7 \%)$

Table 2: Clinical characteristics of patients at the time of diagnosis of PAH, according to NMD pathway status of the BMPR2 mutation.

\begin{tabular}{|c|c|c|c|}
\hline NMD status of BMPR2 mutation & $\begin{array}{l}\text { 'NMD active' (Truncating) } \\
(n=55)\end{array}$ & $\begin{array}{l}\text { 'NMD absent' (Missense) } \\
(n=4 I)\end{array}$ & $P$ value* \\
\hline Age at Diagnosis, yrs & $39.9(36.3-43.5)$ & $30.6(25.9-35.3)$ & 0.004 \\
\hline Gender, female/male & $2.6 / 1$ & $1.7 / 1$ & 0.82 \\
\hline $\mathrm{RAP}, \mathrm{mm} \mathrm{Hg}$ & $11.4(7.4-15.3)$ & $10.5(8.1-13.0)$ & 0.74 \\
\hline Mean PAP, mm Hg & $56.5(51.6-6 \mid .5)$ & $60.0(54.9-65.2)$ & 0.42 \\
\hline PCWP, $\mathrm{mm} \mathrm{Hg}$ & $11.1(8.5-13.6)$ & $10.3(8.4-12.2)$ & 0.74 \\
\hline $\mathrm{Cl}, \mathrm{L} / \mathrm{min} / \mathrm{m}^{2}$ & $1.8(1.5-2.1)$ & $1.9(1.8-2.1)$ & 0.16 \\
\hline PVR, mm Hg/L/min & $18.4(11.4-25.3)$ & $17.9(12.7-23.1)$ & 0.78 \\
\hline $\mathrm{Sv}_{\mathrm{O} 2}, \%$ & $58.6(51.2-65.3)$ & $57.3(52.8-61.7)$ & 0.19 \\
\hline
\end{tabular}

* All $\mathrm{P}$ values calculated using Mann-Whitney $\mathrm{U}$ test.

† $95 \%$ Confidence Interval values calculated using univariable ANOVA

Definition of abbreviations: $\mathrm{Cl}=$ cardiac index; $\mathrm{PAH}$ = pulmonary arterial hypertension; $\mathrm{PCWP}=$ pulmonary capillary wedge pressure; $\mathrm{PAP}=$

pulmonary artery pressure; $\mathrm{PVR}=$ pulmonary vascular resistance; $\mathrm{RAP}=$ right atrial pressure; $\mathrm{Sv}_{\mathrm{O}_{2}}=$ mixed venous oxygen saturation. 


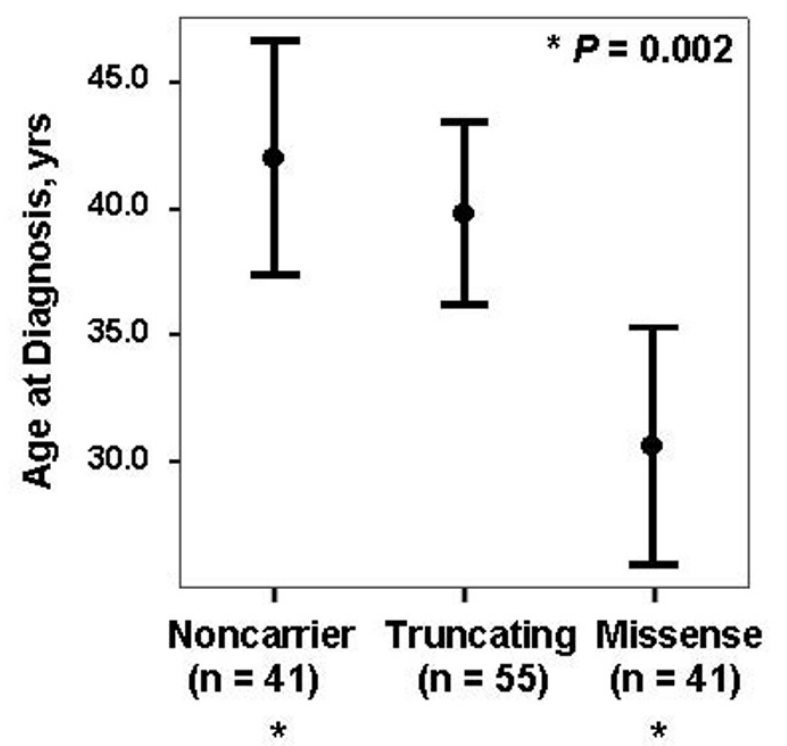

Figure 3

Age at diagnosis of PAH: comparison of noncarriers, patients carrying a truncating BMPR2 mutation, and patients carrying a missense BMPR2 mutation. Age at diagnosis is no different between noncarriers and patients carrying a truncating BMPR2 mutation. There is a significant difference in age at diagnosis between noncarriers and patients carrying a missense BMPR2 mutation (*, $P=0.002$ ). Values represent mean age at diagnosis; error bars represent $95 \%$ confidence intervals.

mutation groups were females diagnosed during or within one year of pregnancy.

\section{Discussion}

We studied 169 patients with HPAH and IPAH with and without germline BMPR2 mutations, all treated at centers in the United States according to the best standard of care available. Our results support the concept that patients who are carriers of a $B M P R 2$ mutation have more severe disease than noncarriers. Specifically, our results support the findings of other investigative groups that patients with a BMPR2 mutation presented at a younger age and had a shorter time to death or lung transplantation, while not supporting the conclusion that carriers have worse hemodynamics at the time of diagnosis $[11,12]$. However, we found that this is only true among females and that patients with missense mutations have more severe disease than patients with truncating mutations (Figures 1, 3, $4,5)$. Significant differences in severity among those with a missense mutation are characterized by a younger age at diagnosis, younger age at death, and a shorter survival from diagnosis to death or transplant. Because these are well described indicators of disease severity, our findings

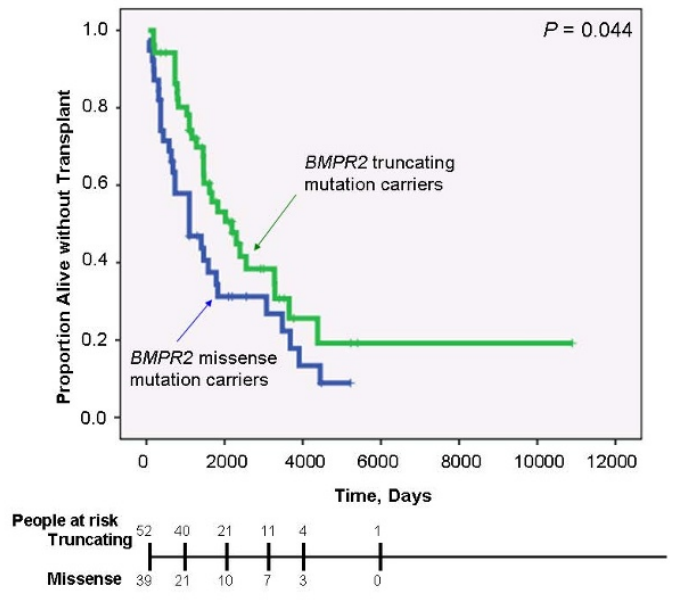

Figure 4

Outcome of BMPR2 mutation carriers with HPAH: truncating versus missense mutation carriers. Survival measured as time to death or lung transplantation, compared between the two groups. Survival is shorter among carriers with a missense mutation (log rank test, $P=0.044$ ).

suggest that in HPAH whether or not a BMPR2 mutation results in a stable (missense mutation) or unstable (truncating mutation) mRNA transcript is a critical modifying variable of severity $[12,25,26]$. As a result, therapies for treatment and prevention directed specifically at BMPR2 pathway defects may need to vary according to the type of mutation. For example, efforts are underway to identify drugs that would increase BMPR2 gene expression as a method of treatment and perhaps disease prevention. For carriers of an unstable mRNA transcript due to a truncat-

Penetrance According to Age and Genotype

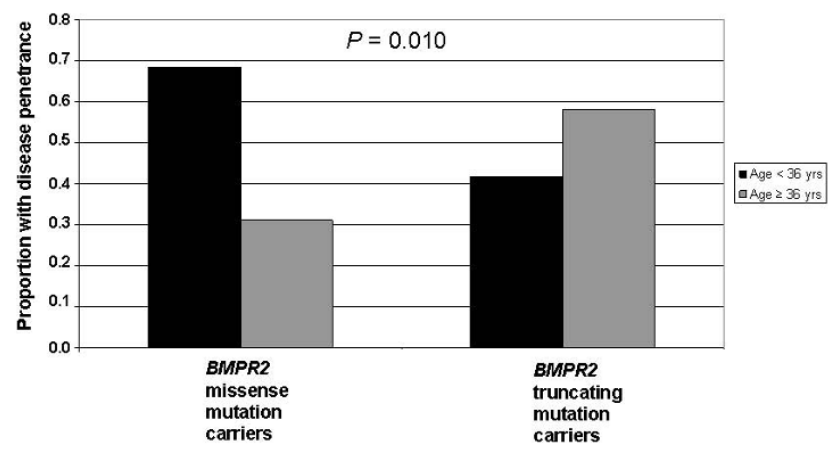

Figure 5

Penetrance of PAH as a function of age. Statistically significant difference in penetrance according to genotype and age in the comparison of the missense mutation group versus the truncating mutation group $(P=0.0 \mathrm{I})$. 
ing mutation, such therapies that directly increase BMPR2 expression might safely and effectively prevent or treat disease by increasing expression by the normal allele without changing the mutated allele's product (because it is inherently unstable). However, such a strategy could be detrimental in carriers of a mutation that produces a stable mRNA transcript associated with more severe disease, since increasing BMPR2 expression could increase both normal allele and mutant allele expression. The effort to tailor therapies to the type of gene mutation is consistent with efforts currently being pursued in the study of other genetic diseases, such as cystic fibrosis [27].

The functional impact of specific BMPR2 mutations has been incompletely investigated to date, with variable consequences for signaling activity reported $[28,29]$. Normally, BMPs regulate growth, differentiation, and apoptosis via an intracellular signaling cascade mediated via cytoplasmic signaling proteins known as Smads, as well as via Smad-independent pathways. The Smad family of proteins are responsible for transforming growth factor $\beta$ receptor signaling to regulate gene expression [30]. There is a delicate balance of Smad signaling among the transforming growth factor $\beta$ receptors, and disruption of this balance may promote a milieu amenable to the development of pulmonary vascular disease among susceptible subjects [7]. This study supports previous suggestions that truncating and missense BMPR2 mutations may differentially impact BMP pathway signaling, and further suggests that they do so via their differential activation of NMD [31]. NMD is a mechanism by which mRNA molecules produced by truncating mutations are detected and eliminated by the cell, resulting in no functional protein from the mutated allele. This produces a haploinsufficient effect, which magnifies the importance of the wild-type allele in protein production. Consistent with this, we recently found that the level of expression of wild-type BMPR2 allele is a critical factor in the pathogenesis of HPAH caused by truncating mutations [32]. In contrast, missense mutations resistant to NMD destruction may produce a mutant protein product with deleterious effects that impair or completely block the activity of the remaining normal allele. This may create a 'dominant negative effect', which affects the phenotype more severely (Figure 1) [16,22].

The comparison of a truncating versus missense mutation may be particularly important for BMP pathway signaling, which is dependent upon appropriate heterodimerization of BMPR2 with type 1 BMP receptors to activate the signaling cascade. Correct signaling requires the proper stoichiometric balance between receptors [33]. While this balance is likely perturbed by most types of BMPR2 mutations, it can be exaggerated by missense mutations, which cause dysfunctional heterodimers that exert dominant negative effects on BMP signaling [34]. Thus, as reported in other genetic diseases, the activation or inactivation of the NMD pathway may profoundly affect the variability of disease phenotypes in HPAH, and our findings support this concept $[16,35]$. From a therapeutic perspective, manipulation of the NMD surveillance pathway specific to a particular BMPR2 mutation might promote a less severe phenotype by changing a dominant negative effect to haploinsufficiency [36].

This study demonstrates how different variations within the same gene may influence disease, although several areas of potential criticism exist. First, because the incidence of HPAH is very low ( 1 case per 1 million adults) and this study requires the creation of cell lines from each patient, like other studies of rare diseases the overall number of patients enrolled is relatively small and the collection of a replicate cohort will take many years [37]. Such an endeavor will require a multicenter collaborative effort, and the design and implementation of that investigation in an independent study population is currently underway. Second, it is possible that the 22 patients with familial PAH excluded from analysis because they did not carry a detectable BMPR2 mutation do carry mutations in unexplored parts of the BMPR2 gene (or that they have inappropriately been categorized as having a familial disease but are in fact patients with IPAH). However, our use of genomic DNA and transcript sequencing and MLPA analysis provided the most comprehensive screen for BMPR2 mutations described to date [20]. Third, because not every truncating genetic mutation predicted to activate the NMD pathway based upon the genotype truly activates NMD (rarely, some do not), cell line testing is required for confirmation as performed in this study. For this reason, subjects were divided for analysis according to the results of cell line testing for NMD pathway activity, not according to predicted activity (in fact, 7 of 96 subjects with cell lines tested for NMD had mutations predicted to result in activation of the NMD pathway that upon testing were not 'NMD active'). Regardless, the key issue is whether or not the mutation is capable of activating the NMD pathway or not, and the vast majority of truncating mutations do activate this pathway. Finally, the absence of significant differences in cardiovascular parameters at diagnosis in this study suggests that additional factors participate in the generation of phenotype, especially highlighting the importance of right heart adaptation to pulmonary vascular disease and the relatively small degree to which we currently understand this process at the cellular, molecular and clinical levels [38]. Efforts are underway to determine whether the response to acute vasodilator testing at the time of diagnosis is different between patients with truncating and missense mutations, as response data may predict survival. [39] 
In conclusion, this study supports previous studies showing increased severity among patients with a BMPR2 mutation compared to those who are noncarriers, but interestingly this is only true for females and for carriers of a certain type of mutation. When we classify mutations according to if they activate (truncating mutations) or escape (missense mutations) NMD destruction of their mutant transcript we see a significant difference in severity between these groups. This supports the concept that missense mutations that escape NMD result in stable transcripts that may produce proteins that are qualitatively different. Their resultant dominant negative effects on BMP signaling make missense mutations more detrimental than truncating mutations, which cause haploinsufficiency. The haploinsufficient effects of truncating mutations occur because the total BMPR2 protein levels are quantitatively reduced to the level of expression of the single wild-type allele. These findings emphasize the need to further explore the manner in which different subclasses of BMPR2 mutations influence phenotype in PAH, as well as the potential role of the wild-type allele.

\section{Competing interests}

The authors declare that they have no competing interests.

\section{Authors' contributions}

EA provided scientific design, wrote the manuscript, performed data management, performed statistical analyses, and participated in the clinical field and laboratory work. JC, RH, CP, KS performed laboratory analyses, data management, and scientific review. LW performed clinical field work, data management and manuscript review. IR and JN contributed to project scientific oversight, and provided clinical field work and manuscript review. JP and JL had primary responsibility for scientific design, scientific oversight of this and related projects, and manuscript review.

\section{Acknowledgements}

The authors thank all of the families participating in this study; without them, this research would not be possible. We also thank Lora Hedges, Cindy Holladay, Melissa Prince, and Keri Hemingway for their tireless efforts in the study of patients and families impacted by PAH. Finally, we thank the National Institutes of Health, Vanderbilt University General Clinical Research Center (GCRC), the Vanderbilt Clinical Research Scholars program, and the Turner-Hazinski Research Fund of the Department of Pediatrics at Vanderbilt University School of Medicine/Monroe Carell Jr. Children's Hospital for study support (POI HL072058, GCRC RR000095, and KI2 RRI 7697).

\section{References}

I. Runo JR, Loyd JE: Primary pulmonary hypertension. The Lancet 2003, 36 I(9368): I533-I544.

2. Machado R, Chung W, Eickelberg O, Elliot G, Hanaoka M, Geraci M, Loyd J, Newman J, Phillips J, Soubrier F, et al.: Genetics and genomics of pulmonary arterial hypertension. J Am Coll Cardiol 2009.

3. Simonneau G, Galie N, Rubin LJ, Langleben D, Seeger W, Domenighetti G, Gibbs S, Lebrec D, Speich R, Beghetti M, et al.: Clin- ical classification of pulmonary hypertension. I Am Coll Cardiol 2004, 43 (I 2 Suppl S):5S- I2S.

4. Deng Z, Morse JH, Slager SL, Cuervo N, Moore KJ, Venetos G, Kalachikov S, Cayanis E, Fischer SG, Barst RJ, et al: Familial primary pulmonary hypertension (gene PPHI) is caused by mutations in the bone morphogenetic protein receptor-II gene. Am J Hum Genet 2000, 67(3):737-744.

5. Lane KB, Blackwell TR, Runo J, Wheeler L, Phillips JA 3rd, Loyd JE: Aberrant signal transduction in pulmonary hypertension. Chest 2005, I 28(6 Suppl):564S-565S.

6. Newman JH, Wheeler L, Lane KB, Loyd E, Gaddipati R, Phillips JA 3rd, Loyd JE: Mutation in the gene for bone morphogenetic protein receptor II as a cause of primary pulmonary hypertension in a large kindred. N Engl J Med 200I, 345(5):3 I9-324.

7. Newman JH, Phillips JA 3rd, Loyd JE: Narrative review: the enigma of pulmonary arterial hypertension: new insights from genetic studies. Ann Intern Med 2008, I 48(4):278-283.

8. Gaine SP, Rubin LJ: Primary pulmonary hypertension. Lancet 1998, 352(9 | 29):7| 9-725.

9. Rich S, Dantzker DR, Ayres SM, Bergofsky EH, Brundage BH, Detre KM, Fishman AP, Goldring RM, Groves BM, Koerner SK, et al:: Primary pulmonary hypertension. A national prospective study. Ann Intern Med 1987, 107(2):216-223.

10. Elliott CG, Glissmeyer EW, Havlena GT, Carlquist J, McKinney JT, Rich S, McGoon MD, Scholand MB, Kim M, Jensen RL, et al.: Relationship of BMPR2 Mutations to Vasoreactivity in Pulmonary Arterial Hypertension. Circulation 2006, I I3(2I):2509-25I5.

II. Rosenzweig EB, Morse JH, Knowles JA, Chada KK, Khan AM, Roberts KE, McElroy JJ, Juskiw NK, Mallory NC, Rich S, et al.: Clinical implications of determining BMPR2 mutation status in a large cohort of children and adults with pulmonary arterial hypertension. J Heart Lung Transplant 2008, 27(6):668-674.

12. Sztrymf B, Coulet F, Girerd B, Yaici A, Jais X, Sitbon O, Montani D, Souza R, Simonneau G, Soubrier F, et al:: Clinical Outcomes of Pulmonary Arterial Hypertension in Carriers of BMPR2 Mutation. Am J Respir Crit Care Med 2008.

13. Sztrymf B, Yaici A, Girerd B, Humbert M: Genes and pulmonary arterial hypertension. Respiration 2007, 74(2): $123-132$.

14. Neu-Yilik G, Kulozik AE: NMD: multitasking between mRNA surveillance and modulation of gene expression. Adv Genet 2008, 62: 185-243.

15. Noensie EN, Dietz HC: A strategy for disease gene identification through nonsense-mediated mRNA decay inhibition. Nat Biotechnol 200I, 19(5):434-439.

16. Khajavi M, Inoue K, Lupski JR: Nonsense-mediated mRNA decay modulates clinical outcome of genetic disease. Eur J Hum Genet 2006, 14(10): 1074-108I.

17. Boyle MP: Strategies for identifying modifier genes in cystic fibrosis. Proc Am Thorac Soc 2007, 4(I):52-57.

18. Loyd JE, Primm RK, Newman JH: Familial primary pulmonary hypertension: clinical patterns. Am Rev Respir Dis 1984, I 29(I): 194-197.

19. McGoon M, Gutterman D, Steen V, Barst R, McCrory DC, Fortin TA, Loyd JE: Screening, early detection, and diagnosis of pulmonary arterial hypertension: ACCP evidence-based clinical practice guidelines. Chest 2004, I 26(I Suppl): I4S-34S.

20. Cogan JD, Pauciulo MW, Batchman AP, Prince MA, Robbins IM, Hedges LK, Stanton KC, Wheeler LA, Phillips lii JA, Loyd JE, et al:: High Frequency of BMPR2 Exonic Deletions/Duplications in Familial Pulmonary Arterial Hypertension. Am J Respir Crit Care Med 2006.

21. Cogan JD, Vnencak-Jones CL, Phillips JA 3rd, Lane KB, Wheeler LA, Robbins IM, Garrison G, Hedges LK, Loyd JE: Gross BMPR2 gene rearrangements constitute a new cause for primary pulmonary hypertension. Genet Med 2005, 7(3):169-174.

22. Machado RD, Aldred MA, James V, Harrison RE, Patel B, Schwalbe EC, Gruenig E, Janssen B, Koehler R, Seeger W, et al: Mutations of the TGF-beta type II receptor BMPR2 in pulmonary arterial hypertension. Hum Mutat 2006, 27(2): $121-132$.

23. Aldred MA, Machado RD, James V, Morrell NW, Trembath RC: Characterization of the BMPR2 5 '-untranslated Region and a Novel Mutation in Pulmonary Hypertension. Am J Respir Crit Care Med 2007.

24. Said SI: Mediators and modulators of pulmonary arterial hypertension. Am J Physiol Lung Cell Mol Physiol 2006. 
25. Humbert M, Sitbon O, Simonneau G: Treatment of pulmonary arterial hypertension. N Engl J Med 2004, 35 I ( I 4): I 425- I 436.

26. Montani D, Souza R, Binkert C, Fischli W, Simonneau G, Clozel M, Humbert M: Endothelin-I/endothelin-3 ratio: a potential prognostic factor of pulmonary arterial hypertension. Chest 2007, | 3 I (I): $|0|-108$.

27. Krasnov KV, Tzetis M, Cheng J, Guggino WB, Cutting GR: Localization studies of rare missense mutations in cystic fibrosis transmembrane conductance regulator (CFTR) facilitate interpretation of genotype-phenotype relationships. Hum Mutat 2008, 29(II): 1364-1372.

28. Rudarakanchana N, Flanagan JA, Chen H, Upton PD, Machado R, Patel $D$, Trembath RC, Morrell NW: Functional analysis of bone morphogenetic protein type II receptor mutations underlying primary pulmonary hypertension. Hum Mol Genet 2002, II(I3): I517-1525.

29. Nishihara A, Watabe T, Imamura T, Miyazono K: Functional heterogeneity of bone morphogenetic protein receptor-II mutants found in patients with primary pulmonary hypertension. Mol Biol Cell 2002, I3(9):3055-3063.

30. Morrell NW, Adnot S, Archer SL, Dupuis J, Jones PL, MacLean MR, McMurtry IF, Stenmark KR, Thistlethwaite PA, Weissmann N, et al.: Cellular and molecular basis of pulmonary arterial hypertension. J Am Coll Cardiol 2009, 54(I Suppl):S20-3 I.

31. Machado RD, Pauciulo MW, Thomson JR, Lane KB, Morgan NV Wheeler L, Phillips JA 3rd, Newman J, Williams D, Galie N, et al: BMPR2 haploinsufficiency as the inherited molecular mechanism for primary pulmonary hypertension. Am J Hum Genet 200I, 68(I):92-102.

32. Hamid R, Cogan JD, Hedges LK, Austin E, Phillips JA 3rd, Newman JH, Loyd JE: Penetrance of pulmonary arterial hypertension is modulated by the expression of normal BMPR2 allele. Hum Mutat 2009, 30(4):649-654.

33. Nasim MT, Ghouri A, Patel B, James V, Rudarakanchana N, Morrell NW, Trembath RC: Stoichiometric imbalance in the receptor complex contributes to dysfunctional BMPR-II mediated signalling in pulmonary arterial hypertension. Hum Mol Genet 2008, I7(II): 1683-1694.

34. Sobolewski A, Rudarakanchana N, Upton PD, Yang J, Crilley TK, Trembath RC, Morrell NW: Failure of bone morphogenetic protein receptor trafficking in pulmonary arterial hypertension: potential for rescue. Hum Mol Genet 2008 , I7(20):3180-3190.

35. Kuzmiak HA, Maquat LE: Applying nonsense-mediated mRNA decay research to the clinic: progress and challenges. Trends Mol Med 2006, I2(7):306-316.

36. Shariat N, Ryther RC, Phillips JA 3rd, Robinson IC, Patton JG: Rescue of Pituitary Function in a Mouse Model of Isolated Growth Hormone Deficiency Type II by RNA Interference. Endocrinology 2008, I 49(2):580-586.

37. Humbert M, Sitbon O, Chaouat A, Bertocchi M, Habib G, Gressin V, Yaici A, Weitzenblum E, Cordier JF, Chabot F, et al.: Pulmonary arterial hypertension in France: results from a national registry. Am J Respir Crit Care Med 2006, I 73(9): 1023-1030.

38. Bogaard HJ, Abe K, Vonk Noordegraaf A, Voelkel NF: The right ventricle under pressure: cellular and molecular mechanisms of right-heart failure in pulmonary hypertension. Chest 2009, I35(3):794-804.

39. Sitbon O, Humbert M, Jais X, loos V, Hamid AM, Provencher S, Garcia $G$, Parent $F$, Herve $P$, Simonneau G: Long-term response to calcium channel blockers in idiopathic pulmonary arterial hypertension. Circulation 2005, I I I(23):3 I05-3 I II.

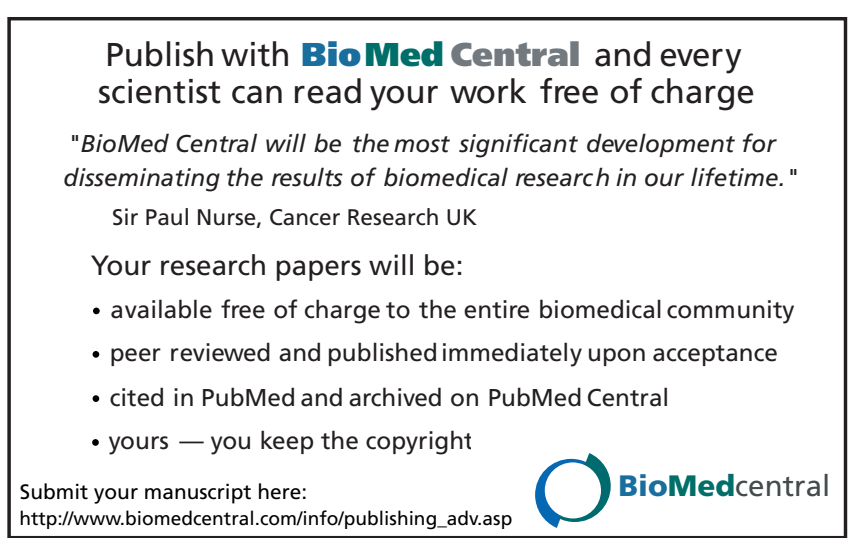

